\title{
ON THE SIZE OF DIOPHANTINE $M$-TUPLES FOR LINEAR POLYNOMIALS
}

\author{
A. FILIPIN AND A. JURASIĆ
}

Received 04 February, 2015

\begin{abstract}
In this paper we prove that there does not exist a set with more than 16 nonzero polynomials in $\mathbb{K}[X]$, where $\mathbb{K}$ is any field of characteristic 0 , such that the product of any two of them increased by a linear polynomial $n \in \mathbb{K}[X]$ is a square of a polynomial from $\mathbb{K}[X]$.
\end{abstract}

2010 Mathematics Subject Classification: 11C08; 11D99

Keywords: Diophantine $m$-tuples, polynomials

\section{INTRODUCTION}

Diophantus of Alexandria [1] was the first who studied the problem of finding sets with the property that the product of any two of its distinct elements increased by 1 is a perfect square. Such a set consisting of $m$ elements is therefore called a Diophantine $m$-tuple. Diophantus found the first Diophantine quadruple consisting of rational numbers $\left\{\frac{1}{16}, \frac{33}{16}, \frac{17}{4}, \frac{105}{16}\right\}$, while the first Diophantine quadruple of integers, the set $\{1,3,8,120\}$, was found by Fermat. In the case of rational numbers no upper bound for the size of such sets is known. In integer case which is the most studied, Dujella [4] proved that there does not exist a Diophantine sextuple and there are only finitely many Diophantine quintuples. The folklore conjecture is that there does not exist a Diophantine quintuple over the integers.

Many generalizations of this problem were also considered, for example by adding a fixed integer $n$ instead of 1 , looking at $k$ th powers instead of squares, or considering the problem over other domains than $\mathbb{Z}$ or $\mathbb{Q}$.

Definition 1. Let $m \geq 2, k \geq 2$ and let $R$ be a commutative ring with 1 . Let $n \in R$ be a nonzero element and let $\left\{a_{1}, \ldots, a_{m}\right\}$ be a set of $m$ distinct nonzero elements from $R$ such that $a_{i} a_{j}+n$ is a $k$ th power of an element of $R$ for $1 \leq i<j \leq m$. The set $\left\{a_{1}, \ldots, a_{m}\right\}$ is called a $k$ th power Diophantine $m$-tuple with the property $D(n)$ or simply a $k$ th power $D(n)$ - $m$-tuple in $R$.

The authors were supported by Croatian Science Foundation, Grant No. 6422. The second author was also supported by the University of Rijeka Research Grant No. 13.14.1.2.02. 
The first such set, a second power $D(256)$-quadruple $\{1,33,68,105\}$, was found by Diophantus [1]. It is interesting to find upper bounds for the number of elements of such sets. Dujella $[2,3]$ found such bounds for the integer case and for $k=2$. For other similar results see $[5,6,9,12]$.

The first polynomial variant of the above problem was studied by Jones $[13,14]$ for $R=\mathbb{Z}[X], k=2$ and $n=1$. In this case, Dujella and Fuchs [6] proved that there does not exist a second power Diophantine quintuple. Dujella and Luca [11] considered the case $n=1, k \geq 3$ and $R=\mathbb{K}[X]$, where $\mathbb{K}$ is an algebraically closed field of characteristic 0. Using many results from [11], Dujella and Jurasić [9] proved that there does not exist a second power Diophantine 8-tuple in $\mathbb{K}[X]$ for $n=1$. There were also considered other variants of such a polynomial problem. Dujella and Fuchs, jointly with Tichy [7] and later with Walsh [8], considered the case $R=\mathbb{Z}[X], k=2$ and $n$ is a linear polynomial from $\mathbb{Z}[X]$. They proved that in this case $m \leq 12$. Jurasić [15] proved that $m \leq 98$ for $n$ a quadratic polynomial in $\mathbb{Z}[X]$.

We will consider the case $R=\mathbb{K}[X]$, where $\mathbb{K}$ is any field of characteristic $0, k=2$ and $n$ is a linear polynomial from $\mathbb{K}[X]$. Without loss of generality we assume that $\mathbb{K}$ is algebraically closed. If we omit the condition that $\mathbb{K}$ is a field of characteristic 0 , then we could not obtain some results where the factorisation of a polynomial is considered. For brevity, instead of second power $D(n)-m$-tuple in $\mathbb{K}[X]$, from now on we shall refer to a polynomial $D(n)$ - $m$-tuple. Observe that, at most one polynomial $a_{i}$ for $i \in\{1, \ldots, m\}$ in such a polynomial $D(n)$-m-tuple is constant. Otherwise, we would have two different constants $a$ and $b$ for which $a b+n=r^{2}$, where $r \in \mathbb{K}[X]$. This is not possible, because then $\operatorname{deg}(n)=1=2 \operatorname{deg}(r)$.

An improper $k$ th power $D(n)$-m-tuple in $R$ is an $m$-tuple with the property from Definition 1, but with relaxed condition that its elements need not be distinct and need not be nonzero. For linear $n$, we cannot have 0 in an improper polynomial $D(n)$ - $m$-tuple. Let us assume that there exists a non-constant polynomial $a$ such that $a^{2}+n=r^{2}$, for some $r \in \mathbb{K}[X]$. Then $\operatorname{deg}(a)=\operatorname{deg}(r) \geq 1$ and $\operatorname{deg}(n)=$ $\operatorname{deg}(r-a)+\operatorname{deg}(r+a)$. This is not possible if $\operatorname{deg}(a) \geq 2$ but, for example, we have $(X+3)^{2}-4 X-8=(X+1)^{2}$. So, in an improper polynomial $D(n)$-m-tuple we can have infinitely many equal linear polynomials. In the rest of the paper we consider only proper $D(n)$ - $m$-tuples, described in Definition 1 . We have the following theorem.

Theorem 1. There are at most 16 elements in a polynomial $D(n)$-m-tuple for a linear polynomial $n$, i.e.

$$
m \leq 16 .
$$

In order to prove Theorem 1, we follow the strategy used in $[7,8]$ for linear $n$ and in [15] for quadratic $n$. In those papers the ring $\mathbb{Z}[X]$ was considered, using the relation "<" between its elements. Instead of that, in $\mathbb{K}[X]$ we have to use the relation " $\leq$ " between the degrees of its elements. The paper is organized as follows. In Section 2 
we estimate the number of polynomials with given degree $k$ in a polynomial $D(n)$ $m$-tuple and consider separate cases depending on $k$. In Section 3, we adapt the gap principle for the degrees of the elements of a polynomial $D(n)$-quadruple, proved in [7] for the ring $\mathbb{Z}[X]$, to $\mathbb{K}[X]$. Using the bounds from Section 2 and combining the gap principle with an upper bound for the degree of the largest element in a polynomial $D(n)$-quadruple, obtained in [8] and also valid in $\mathbb{K}[X]$, in Section 4 we give the proof of Theorem 1 .

\section{Sets With Polynomials Of EQUAL DEgReE}

Let $L_{k}$ be the number of polynomials of degree $k$ from $\mathbb{K}[X]$ in a polynomial $D(n)$ - $m$-tuple for linear $n$. The first step which leads us to the proof of Theorem 1 is to estimate the numbers $L_{k}$ for $k \geq 0$. We already proved that

$$
L_{0} \leq 1 \text {. }
$$

The following lemma, which is [7, Lemma 1], plays the key role in our proofs. It is proved for polynomials with integer coefficients, but the proof is obtained using only algebraic manipulations so it holds in $\mathbb{K}[X]$ also.

Lemma 1. Let $\{a, b, c\}$ be a polynomial $D(n)$-triple in $\mathbb{K}[X]$ and let

$$
a b+n=r^{2}, a c+n=s^{2}, b c+n=t^{2},
$$

for some $r, s, t \in \mathbb{K}[X]$. Then there exist polynomials e, $u, v, w \in \mathbb{K}[X]$ such that

$$
a e+n^{2}=u^{2}, b e+n^{2}=v^{2}, c e+n^{2}=w^{2} .
$$

More precisely,

$$
\begin{gathered}
e=n(a+b+c)+2 a b c-2 r s t, \\
u=a t-r s, \quad v=b s-r t, w=c r-s t
\end{gathered}
$$

and

$$
c=a+b+\frac{e}{n}+\frac{2}{n^{2}}(a b e+r u v) .
$$

We also define

$$
\bar{e}=n(a+b+c)+2 a b c+2 r s t
$$

and we get

$$
e \cdot \bar{e}=n^{2}(c-a-b-2 r)(c-a-b+2 r) .
$$

Also, from (2.3), using (2.1) and (2.4), we get

$$
e=n(a+b-c)+2 r w,
$$

$e=n(a-b+c)+2 s v$ and $e=n(-a+b+c)+2 t u$.

Let $\operatorname{deg}(n)=1$ and $\operatorname{deg}(a)=\operatorname{deg}(b)=\operatorname{deg}(c)=k \geq 1$. From (2.1) we obtain $\operatorname{deg}(r)=\operatorname{deg}(s)=\operatorname{deg}(t)=k \geq 1$. Let $A, B, C, R, S, T$ be the leading coefficients 
of the polynomials $a, b, c, r, s, t$, respectively. Then, from (2.1), we have $A B=R^{2}$, $A C=S^{2}$ and $B C=T^{2}$ so $A B C= \pm R S T$. Let us consider both cases.

1.) If $A B C=R S T$, from (2.6) we have $\operatorname{deg}(\bar{e})=3 k$ and then, from (2.7),

$$
\operatorname{deg}(e) \leq 2-k
$$

2.) If $A B C=-R S T$, from (2.3) we conclude

$$
\operatorname{deg}(e)=3 k,
$$

and then, from $(2.7), \operatorname{deg}(\bar{e}) \leq 2-k$.

In order to bound the number $L_{k}$ for $k \geq 1$, we are interested to find the number of possible $c$-s, for fixed $a$ and $b$, such that (2.1) holds. The first step is finding all possible $e$-s from Lemma 1. The trivial situation in 1.) is $e=0$. Then, from (2.7), we obtain

$$
c_{ \pm}=a+b \pm 2 r
$$

Analogue situation in 2.) is $\bar{e}=0$. Beside those trivial situations we suppose that $\operatorname{deg}(e) \geq 0$ and $\operatorname{deg}(\bar{e}) \geq 0$.

Polynomial $D(n)$-triples can be classified as regular or irregular, depending on whether they satisfy the condition given in the next definition (see e.g. [10]).

Definition 2. A $D(n)$-triple $\{a, b, c\}$ is called regular if it satisfies the condition

$$
(c-b-a)^{2}=4(a b+n) .
$$

Observe that any permutation of $a, b, c$ leaves equation (2.12) invariant. Also, from (2.12), using (2.1), we get (2.11) and we have

$$
a c_{ \pm}+n=(a \pm r)^{2}, b c_{ \pm}+n=(b \pm r)^{2} .
$$

Moreover, a semi-regular $D(n)$-quadruple is one which contains a regular triple, and a twice semi-regular $D(n)$-quadruple is one that contains two regular triples.

Let us consider a polynomial $D(n)$-triple $\{a, b, c\}$ in the two following lemmas. We will use the divisibility of polynomials in $\mathbb{K}[X]$. A polynomial $p$ divides a polynomial $q$ if there exists a polynomial $m$ from $\mathbb{K}[X]$ such that $q=m p$. This situation will be denoted by $p \mid q$.

Lemma 2. At most one element of a polynomial $D(n)$-triple $\{a, b, c\}$ is divisible by $n$.

Proof. Without loss of generality, let $a$ and $b$ be divisible by $n$. Then, from (2.1), it follows that $n \mid r$. Hence, $n^{2} \mid n$, a contradiction.

In the following lemma, we adapt the important result from [8] for $\mathbb{K}[X]$.

Lemma 3. Let $\{a, b\}$ be a polynomial $D(n)$-pair with $\operatorname{deg}(a)=\operatorname{deg}(b)=k \geq 1$ and such that $a b+n=r^{2}$, where $r \in \mathbb{K}[X]$. Let ae $+n^{2}=u^{2}$, be $+n^{2}=v^{2}$, where $u, v, e \in \mathbb{K}[X]$ and $\operatorname{deg}(e) \geq 0$. Then for each such e there is at most one $c \in \mathbb{K}[X]$, with $\operatorname{deg}(c)=k$, such that $\{a, b, c\}$ is a polynomial $D(n)$-triple. 
Proof. Suppose that $\{a, b\}$ is a polynomial $D(n)$-pair such that $a b+n=r^{2}$, where $r \in \mathbb{K}[X]$. From $a e+n^{2}=u^{2}$ and $b e+n^{2}=v^{2}$, where $u, v \in \mathbb{K}[X]$, and from the fact that $\mathbb{K}$ is an algebraically closed field, we conclude that there are at most two $u$-s and at most two $v$-s. Namely, we have $\pm u$ and $\pm v$. Then, from (2.5), we obtain two possible $c$-s:

$$
c_{ \pm}=a+b+\frac{e}{n}+\frac{2}{n^{2}}(a b e \pm r u v)
$$

From this, we get

$$
c_{+} \cdot c_{-}=b^{2}+a(a-2 b)+\frac{e^{2}}{n^{2}}-\frac{2 a e}{n}-\frac{2 b e}{n}-4 n .
$$

If $c_{+} \cdot c_{-} \in \mathbb{K}[X]$, then $\frac{e(e-2 n(a+b))}{n^{2}} \in \mathbb{K}[X]$. From this, we conclude that $n \mid e$. Then, from (2.2), we get that $n|u, n| v$, and further $n^{2} \mid a e$ and $n^{2} \mid b e$. If $n^{2} \nmid e$, then $n \mid a$ and $n \mid b$, which is in contradiction with Lemma 2. Hence, $n^{2} \mid e$. In that case (2.9) is not possible and we must have (2.10). Then, from (2.15), we obtain that

$$
\operatorname{deg}\left(c_{+}\right)+\operatorname{deg}\left(c_{-}\right)=\operatorname{deg}\left(\frac{e^{2}}{n^{2}}\right)=6 k-2 .
$$

Finally, we conclude that one of the polynomials $c_{ \pm}$has a degree $5 k-2$ if the other of them has a degree $k$.

Remark 1. From the proof of Lemma 3 we have that if $n \mid e$, then $n^{2} \mid e$. A polynomial $D(16 X+9)$-triple

$$
\{X, 16 X+8,36 X+20\},
$$

from [7], for which $e=33 X+18$ is an example for which (2.9) holds. But in this case $c_{+} \cdot c_{-} \notin \mathbb{K}[X]$.

\subsection{Linear polynomials}

Let us prove the following proposition.

Proposition 1. $L_{1} \leq 7$.

For the proof of Proposition 1 we use the results from the previous lemmas and the results stated below. Let us fix $a, b \in \mathbb{K}[X]$ such that $\operatorname{deg}(a)=\operatorname{deg}(b)=1$. We are looking at the extensions of $\{a, b\}$ to a polynomial $D(n)$-triple $\{a, b, c\}$ with $\operatorname{deg}(c)=$ 1 and then at the corresponding $e \in \mathbb{K}[X]$ defined by (2.3). From (2.9), we have the first possibility that $\operatorname{deg}(e) \leq 1$ and from (2.10) we have the second possibility that $\operatorname{deg}(e)=3$. Therefore, we have to consider the possibilities that $\operatorname{deg}(e) \in\{0,1,3\}$.

Lemma 4. For a fixed polynomial $D(n)$-pair $\{a, b\}$ with $\operatorname{deg}(a)=\operatorname{deg}(b)=1$ there is at most one $c$ with $\operatorname{deg}(c)=1$ such that $\{a, b, c\}$ is a polynomial $D(n)$-triple and such that the corresponding e, defined by (2.3), is from $\mathbb{K} \backslash\{0\}$. 
Proof. By Lemma 1, there is $u \in \mathbb{K}[X]$ such that $a e+n^{2}=u^{2}$ and $\operatorname{deg}(u)=$ 1. Since $a=A(X-\phi)$, where $\phi \in \mathbb{K}$ and $A \in \mathbb{K} \backslash\{0\}$, we assume without loss of generality that $u-n=\varepsilon_{1}(X-\phi), u+n=\varepsilon_{2}$, where $\varepsilon_{1}, \varepsilon_{2} \in \mathbb{K} \backslash\{0\}$ and $\varepsilon_{1} \varepsilon_{2}=A e$. This implies that

$$
2 n=-\varepsilon_{1} X+\varepsilon_{2}+\varepsilon_{1} \phi .
$$

Assume that, for fixed $a$ and $b$, two distinct $e$-s exist, namely $e$ and $f$, where $f \in \mathbb{K} \backslash\{0\}$ such that Lemma 1 holds. Therefore, af $+n^{2}=u_{1}^{2}$, where $u_{1} \in \mathbb{K}[X]$ and $\operatorname{deg}\left(u_{1}\right)=1$. We conclude that

$$
\begin{aligned}
& u_{1}-n=\varphi_{1}(X-\phi), \\
& u_{1}+n=\varphi_{2}
\end{aligned}
$$

or

$$
\begin{aligned}
& u_{1}-n=\varphi_{1}, \\
& u_{1}+n=\varphi_{2}(X-\phi),
\end{aligned}
$$

where $\varphi_{1} \varphi_{2}=A f$ and $\varphi_{1}, \varphi_{2} \in \mathbb{K} \backslash\{0\}$. Let us first consider the case (2.18). We get $2 n=-\varphi_{1} X+\varphi_{2}+\varphi_{1} \phi$. Hence, from (2.17), $-\varepsilon_{1}=-\varphi_{1}$ and $\varepsilon_{2}+\varepsilon_{1} \phi=\varphi_{2}+\varphi_{1} \phi$. So, we have $\varepsilon_{1}=\varphi_{1}$ and $\varepsilon_{2}-\varphi_{2}=\phi\left(-\varepsilon_{1}+\varphi_{1}\right)$, from which it follows that and $\varepsilon_{2}=\varphi_{2}$. Therefore, $e=f$.

Assume now that (2.19) holds. Then, $2 n=\varphi_{2} X-\varphi_{2} \phi-\varphi_{1}$. Therefore, $-\varepsilon_{1}=\varphi_{2}$ and $\varepsilon_{2}=-\varphi_{1}$. This yields $e=f$. Hence, for fixed $a$ and $b$, there is at most one $e \in \mathbb{K} \backslash\{0\}$. For that $e$, from Lemma 3 , there is at most one $c \in \mathbb{K}[X]$, with $\operatorname{deg}(c)=1$, such that $\{a, b, c\}$ is a polynomial $D(n)$-triple.

Remark 2. Using the proof of Lemma 4, we get a polynomial $D\left(-2 X+\frac{1}{2}\right)$-triple

$$
\left\{X+\frac{\sqrt{2}-10}{49}, 8 X+\frac{64 \sqrt{2}+46}{49}, X(9-4 \sqrt{2})\right\},
$$

for which $e=\frac{36+16 \sqrt{2}}{49}$. Hence, the situation described in Lemma 4 is possible.

Lemma 5. For a fixed polynomial $D(n)$-pair $\{a, b\}$ with $\operatorname{deg}(a)=\operatorname{deg}(b)=1$ there are at most two $c-s$ with $\operatorname{deg}(c)=1$ such that $\{a, b, c\}$ is a polynomial $D(n)$ triple and such that the corresponding e, defined by (2.3), is from $\mathbb{K}[X]$ and $\operatorname{deg}(e)=$ 1.

Proof. From (2.2), we have

$$
c=\frac{(w-n)(w+n)}{e} .
$$

Therefore, $w \pm n=\lambda e$ where $\lambda \in \mathbb{K} \backslash\{0\}$, for at least one of the signs \pm . Inserting that into (2.20), we get

$$
c=\lambda(\lambda e \mp 2 n) \text {. }
$$


Then, from (2.8), it follows that $e(1-2 r \lambda)=n(a+b-c \mp 2 r)$. From Remark 1, we know that if $n \mid e$ then $n^{2} \mid e$, so $n \nmid e$. Therefore,

$$
\begin{gathered}
1-2 r \lambda=\lambda_{1} n, \\
\lambda_{1} e=a+b-c \mp 2 r,
\end{gathered}
$$

where $\lambda, \lambda_{1} \in \mathbb{K} \backslash\{0\}$.

Suppose that for fixed $a$ and $b$ another $e$ exists. We call it $f$, and we suppose that for such $f \in \mathbb{K}[X]$, where $\operatorname{deg}(f)=1$, Lemma 1 holds. For a polynomial $D(n)$-triple $\left\{a, b, c^{\prime}\right\}$, where $\operatorname{deg}\left(c^{\prime}\right)=1$, by Lemma 1 there is $w^{\prime} \in \mathbb{K}[X]$ such that $c^{\prime} f+n^{2}=$ $\left(w^{\prime}\right)^{2}$. Analogously as for $e$, it holds

$$
1-2 r \xi=\xi_{1} n,
$$

where $\xi_{1} f=a+b-c^{\prime} \mp 2 r, w^{\prime} \pm n=\xi f$ (for at least one of the signs \pm ) and $\xi, \xi_{1} \in \mathbb{K} \backslash\{0\}$.

From (2.22) and (2.24), we get $-2 r(\lambda-\xi)=n\left(\lambda_{1}-\xi_{1}\right)$. If $n \mid r$, we obtain a contradiction with (2.22). Therefore, $\lambda=\xi$ and $\lambda_{1}=\xi_{1}$.

By inserting (2.21) into (2.23), we obtain

$$
e=\frac{1}{\lambda^{2}+\lambda_{1}}(a+b \mp 2 r \pm 2 n \lambda) .
$$

Analogously, we get

$$
f=\frac{1}{\xi^{2}+\xi_{1}}(a+b \mp 2 r \pm 2 n \xi) .
$$

Comparing (2.26) and (2.25), we conclude that there is at most one such $f \neq e$, namely one obtained for different combination of signs in (2.25) and (2.26).

Suppose that there is a third $e$, namely $h \in \mathbb{K}[X]$, where $\operatorname{deg}(h)=1$, for which Lemma 1 holds. Analogously we conclude that $h=e$ or $h=f$. For each of polynomials $e$ and $f$, by Lemma 3, we have at most one different linear polynomial $c$, namely $c$ and $c^{\prime}$, such that $\{a, b, c\}$ and $\left\{a, b, c^{\prime}\right\}$ are polynomial $D(n)$-triples.

Remark 3. The situation from the proof of Lemma 5 is possible. Examples for that are the polynomial $D(16 X+9)$-triples (2.16) and $\{X, 16 X+8,100 X+44\}$, for which $f=273 X+126$. In this case $\lambda=\frac{2}{3}$ and $\lambda_{1}=-\frac{1}{3}$. Also, by iserting $X^{2}$ instead of $X$ in those examples, we get examples obtained in [15].

Let us now consider the last possibility, that (2.10) holds, i.e. $\operatorname{deg}(\bar{e}) \leq 1$. Since we assumed that $\operatorname{deg}(\bar{e}) \geq 0$, from (2.7) we conclude that $n \mid e$.

Lemma 6. For a fixed polynomial $D(n)$-pair $\{a, b\}$ with $\operatorname{deg}(a)=\operatorname{deg}(b)=1$ there does not exist $c$ with $\operatorname{deg}(c)=1$ such that $\{a, b, c\}$ is a polynomial $D(n)$-triple and such that the corresponding e, defined by (2.3), is from $\mathbb{K}[X]$ and $\operatorname{deg}(e)=3$. 
Proof. Assume that such an $e$ exists. Since $n \mid e$ then $n^{2} \mid e$, by Remark 1. If we, for fixed $a$ and $b$, have such a triple $\{a, b, c\}$, then $c e+n^{2}=w^{2}$, for $w \in \mathbb{K}[X]$. We conclude that $\operatorname{deg}(w)=2$ and $n \mid w$. Therefore, from the last equation, we get $c e_{1}+1=w_{1}^{2}$, where $e_{1}, w_{1} \in \mathbb{K}[X]$ and $\operatorname{deg}\left(e_{1}\right)=\operatorname{deg}\left(w_{1}\right)=1$. We actually have two possibilities for the polynomial $w_{1}$, namely $\pm w_{1}$.

By dividing (2.8) by $n$, we obtain

$$
c=a+b-n e_{1} \pm 2 r w_{1},
$$

which is actually $c_{ \pm}$given with (2.14), and one of the polynomials $c_{ \pm}$must have a degree equal to 2 . Since $\operatorname{deg}\left(c_{ \pm}\right)+\operatorname{deg}\left(c_{\mp}\right)=4$, we conclude that neither one of the polynomials $c$ obtained in this way has degree equal to 1 .

Now we can estimate the number $L_{1}$.

Proof of Proposition 1. Let $a, b \in \mathbb{K}[X]$ be linear polynomials such that $a b+n=$ $r^{2}$, with $r \in \mathbb{K}[X]$. We want to find the number of possible $D(n)$-triples $\{a, b, c\}$, where $c \in \mathbb{K}[X]$ is also a linear polynomial.

For $e=0$, from (2.11), we get as candidates for $c$ at most two polynomials

$$
c_{1,2}=c_{ \pm}=a+b \pm 2 r .
$$

By Lemma 4 , there can exists another $c$, which is one of the polynomials

$$
c_{3}=a+b+\frac{e_{3}}{n}+\frac{2}{n^{2}}\left(a b e_{3} \pm r u_{3} v_{3}\right),
$$

with $e_{3} \in \mathbb{K} \backslash\{0\}$, for which $a e_{3}+n^{2}=u_{3}^{2}, b e_{3}+n^{2}=v_{3}^{2}$, where $u_{3}, v_{3} \in \mathbb{K}[X]$. By Lemma 5 , as a possible $c$ we obtain at most one of the polynomials

$$
c_{i}=a+b+\frac{e_{i}}{n}+\frac{2}{n^{2}}\left(a b e_{i} \pm r u_{i} v_{i}\right)
$$

for each $i=4,5$, with linear polynomials $e_{i} \in \mathbb{K}[X]$ and with $u_{i}, v_{i} \in \mathbb{K}[X]$ such that $a e_{i}+n^{2}=u_{i}^{2}, b e_{i}+n^{2}=v_{i}^{2}$.

Since there are no other possibilities for $e$, we conclude that a polynomial $D(n)$ tuple which contains polynomials $a$ and $b$, and consists only of linear polynomials, has at most seven elements, namely, $\left\{a, b, c_{1}, c_{2}, c_{3}, c_{4}, c_{5}\right\}$.

Remark 4. The example $a=X-\frac{7}{12}, b=4 X, n=X+\frac{1}{9}, r=2 X-\frac{1}{3}, c_{1}=c_{+}=$ $9 X-\frac{5}{4}$ and $c_{2}=c_{-}=X+\frac{1}{12}$ shows that a twice semi-regular $D(n)$-quadruple of the form $\left\{a, b, c_{1}, c_{2}\right\}$ is possible in the case of linear polynomials so we can not exclude this possibility in the proof of Proposition 1.

\subsection{Quadratic polynomials}

We intend to prove the following proposition.

Proposition 2. $L_{2} \leq 4$. 
The proof of Proposition 2 is based on the constructions from Lemmas 1- 3 and from the following lemmas which deal with a polynomial $D(n)$-triple $\{a, b, c\}$, where $\operatorname{deg}(a)=\operatorname{deg}(b)=\operatorname{deg}(c)=2$. First, we are looking for the possible $e$-s for fixed $a$ and $b$. We have the following possible cases: $\operatorname{deg}(e) \leq 0$, which comes from (2.9), and $\operatorname{deg}(e)=6$, from (2.10). Therefore, we consider the possibilities that $\operatorname{deg}(e)=0$ and $\operatorname{deg}(e)=6$.

Lemma 7. For a fixed polynomial $D(n)$-pair $\{a, b\}$ with $\operatorname{deg}(a)=\operatorname{deg}(b)=2$ there is at most one $c$ with $\operatorname{deg}(c)=2$ such that $\{a, b, c\}$ is a polynomial $D(n)$-triple and such that the corresponding e, defined by (2.3), is from $\mathbb{K} \backslash\{0\}$.

Proof. By Lemma 1, there is $u \in \mathbb{K}[X]$ such that $a e+n^{2}=u^{2}$ and $\operatorname{deg}(u) \leq 1$. Since $a=A\left(X-\phi_{1}\right)\left(X-\phi_{2}\right)$, where $\phi_{1}, \phi_{2} \in \mathbb{K}$ and $A \in \mathbb{K} \backslash\{0\}$, we assume that

$$
\begin{aligned}
& u-n=\varepsilon_{1}\left(X-\phi_{1}\right), \\
& u+n=\varepsilon_{2}\left(X-\phi_{2}\right),
\end{aligned}
$$

where $\varepsilon_{1}, \varepsilon_{2} \in \mathbb{K} \backslash\{0\}$ and $\varepsilon_{1} \varepsilon_{2}=A e$. From that we conclude

$$
2 n=X\left(\varepsilon_{2}-\varepsilon_{1}\right)+\varepsilon_{1} \phi_{1}-\varepsilon_{2} \phi_{2} .
$$

Let, for fixed $a$ and $b$, two distinct $e$-s from $\mathbb{K} \backslash\{0\}$ exist, i.e. there is also $f \in$ $\mathbb{K} \backslash\{0\}$ for which $a f+n^{2}=u_{1}^{2}$, where $u_{1} \in \mathbb{K}[X]$ and $\operatorname{deg}\left(u_{1}\right) \leq 1$. We have

$$
\begin{aligned}
& u_{1}-n=\varphi_{1}\left(X-\phi_{1}\right), \\
& u_{1}+n=\varphi_{2}\left(X-\phi_{2}\right),
\end{aligned}
$$

or

$$
\begin{aligned}
& u_{1}-n=\varphi_{1}\left(X-\phi_{2}\right), \\
& u_{1}+n=\varphi_{2}\left(X-\phi_{1}\right),
\end{aligned}
$$

where $\varphi_{1} \varphi_{2}=A f$ and $\varphi_{1}, \varphi_{2} \in \mathbb{K} \backslash\{0\}$.

From (2.30), we get $2 n=X\left(\varphi_{2}-\varphi_{1}\right)+\varphi_{1} \phi_{1}-\varphi_{2} \phi_{2}$. By comparing that with (2.29), we obtain $\phi_{1}\left(\varepsilon_{1}-\varphi_{1}\right)=\phi_{2}\left(\varepsilon_{2}-\varphi_{2}\right)=\phi_{2}\left(\varepsilon_{1}-\varphi_{1}\right)$, from which it follows that $\phi_{1}=\phi_{2}$ or $\varepsilon_{1}=\varphi_{1}$. If $\phi_{1}=\phi_{2}$ then, from (2.28), we conclude that $n^{2} \mid a$. Further, from (2.1), we get that $n \mid r$, and then, from (2.3), it follows that $n \mid e$, which is not possible. If $\varepsilon_{1}=\varphi_{1}$, then $\varepsilon_{2}=\varphi_{2}$, so $e=f$.

Assume now that (2.31) holds. Then, $2 n=\left(\varphi_{2}-\varphi_{1}\right) X-\varphi_{2} \phi_{1}+\varphi_{1} \phi_{2}$. By comparing that with (2.29), we obtain $\phi_{1}\left(\varepsilon_{1}+\varphi_{2}\right)=\phi_{2}\left(\varepsilon_{2}+\varphi_{1}\right)=\phi_{2}\left(\varepsilon_{1}+\varphi_{2}\right)$, from which $\phi_{1}=\phi_{2}$ or $\varepsilon_{1}=-\varphi_{2}$. For $\phi_{1}=\phi_{2}$ we obtain a contradiction, as in the previous case. If $\varepsilon_{1}=-\varphi_{2}$, then $\varepsilon_{2}=-\varphi_{1}$, so $e=f$. Hence, for fixed $a$ and $b$, there is at most one $e \in \mathbb{K} \backslash\{0\}$ for which Lemma 1 holds. For that $e$, from Lemma 3, there is at most one $c \in \mathbb{K}[X]$ with $\operatorname{deg}(c)=2$, such that $\{a, b, c\}$ is a polynomial $D(n)$-triple. 
We are left with the last possibility, that (2.10) holds, i.e. $\operatorname{deg}(\bar{e}) \leq 0$. Since we assumed that $\operatorname{deg}(\bar{e}) \geq 0$, from (2.7) we conclude that $n^{2} \mid e$.

Lemma 8. For a fixed polynomial $D(n)$-pair $\{a, b\}$ with $\operatorname{deg}(a)=\operatorname{deg}(b)=2$ there does not exist $c$ with $\operatorname{deg}(c)=2$ such that $\{a, b, c\}$ is a polynomial $D(n)$-triple and such that the corresponding $e$, defined by (2.3), is from $\mathbb{K}[X]$ and $\operatorname{deg}(e)=6$.

Proof. Assume, on the contrary that such an $e$ exists. Then $c e+n^{2}=w^{2}$ for some $w \in \mathbb{K}[X]$. We conclude that $\operatorname{deg}(w)=4$ and $n \mid w$. By dividing the last equation by $n^{2}$ we get $c e_{1}+1=w_{1}^{2}$, where $e_{1}, w_{1} \in \mathbb{K}[X], \operatorname{deg}\left(e_{1}\right)=4$ and $\operatorname{deg}\left(w_{1}\right)=3$. The polynomial $w_{1}$ is only determined up to the sign.

Analogously as in the proof of Lemma 6, we obtain (2.27), from which we conclude that one of the polynomials $c_{ \pm}$has a degree equal to 5 . Since $\operatorname{deg}\left(c_{ \pm}\right)+$ $\operatorname{deg}\left(c_{\mp}\right)=10$, we conclude that neither one of polynomials $\pm w_{1} c$ obtained this way has a degree equal to 2 .

Before we estimate the number $L_{2}$ we will exclude one possibility, which exists in the case of linear polynomials.

Lemma 9. Let $a, b \in \mathbb{K}[X]$ such that $\operatorname{deg}(a)=\operatorname{deg}(b)=2$ and $a b+n=r^{2}$. Then the set of the form $\{a, b, a+b+2 r, a+b-2 r\}$, which contains only quadratic polynomials, is not a polynomial D(n)-quadruple.

Proof. Assume that such a set is a polynomial $D(n)$-quadruple. Let us consider the triples $\{a, a+b+2 r, a+b-2 r\}$ and $\{b, a+b+2 r, a+b-2 r\}$. If the first triple is regular, then, from $(2.12)$, we have $(-4 r-a)^{2}=4(a(a+b+2 r)+n)$. From that, $-4 r-a= \pm 2(a+r)$ so $r \mid a$. Then, from (2.1), it follows that $r \mid n$, which is not possible, because $\operatorname{deg}(r)=2$ and $\operatorname{deg}(n)=1$. The second case is analogous. Therefore, neither one of those triples is regular.

By Lemma 7, for the pair $\{a+b+2 r, a+b-2 r\}$, there is at most one $e \in \mathbb{K} \backslash\{0\}$ such that Lemma 1 holds. Since no other $e$-s exist for that pair, it holds $a=b$ which is not possible.

Remark 5. The example $a=X^{2}+X+\frac{17}{36}, b=X^{2}+2 X-\frac{1}{9}, n=-\frac{2}{3} X+\frac{1}{18}$, $r=X^{2}+\frac{3}{2} X+\frac{1}{18}, a+b+2 r=4 X^{2}+6 X+\frac{17}{36}$ and $a+b-2 r=\frac{1}{4}$ shows that if we omit the condition in Lemma 9 that all polynomials in a set of the form $\{a, b, a+$ $b+2 r, a+b-2 r\}$ are quadratic, then such a $D(n)$-quadruple can exist.

Now we determine the upper bound for $L_{2}$.

Proof of Proposition 2. Let $a, b \in \mathbb{K}[X]$ be quadratic polynomials such that $a b+$ $n=r^{2}$, with $r \in \mathbb{K}[X]$. We look for the number of possible $D(n)$-triples $\{a, b, c\}$, where $c \in \mathbb{K}[X]$ and $c$ is also a quadratic polynomial.

By Lemma 7, there is at most one possibility for $c$, namely $c_{1}$, for which $e \in \mathbb{K} \backslash\{0\}$. For $e=0$ we obtain $c_{2,3}=a+b \pm 2 r$. In Lemma 8 , we excluded the last option 
which comes from Lemma 1 , those that $\operatorname{deg}(e)=6$. Hence, the largest set which we can obtain is of the form $\left\{a, b, c_{1}, c_{2}, c_{3}\right\}$.

By Lemma 9, the set $\left\{a, b, c_{2}, c_{3}\right\}$ is not a polynomial $D(n)$-quadruple. Therefore, the pair $\{a, b\}$ can be extended with at most 2 quadratic polynomials $\left(c_{1}\right.$ and one of the polynomials $c_{2,3}$ ), so $L_{2} \leq 4$.

\subsection{Polynomials of degree $k \geq 3$}

Now we are looking for the upper bound for the number of polynomials of degree $k \geq 3$ in a polynomial $D(n)$-tuple.

Proposition 3. $L_{k} \leq 3$ for $k \geq 3$.

Let $a, b \in \mathbb{K}[X]$ be polynomials of degree $k \geq 3$ such that $a b+n=r^{2}$, with $r \in \mathbb{K}[X]$. We look for the number of possible $c$-s such that $\{a, b, c\}$, where $c \in \mathbb{K}[X]$ and $\operatorname{deg}(c)=k$ is a polynomial $D(n)$-triple. First, we look for possible $e$-s such that Lemma 1 holds. From (2.9), $\operatorname{deg}(e) \leq-1$, and from $(2.10), \operatorname{deg}(\bar{e}) \leq-1$. Therefore, $e=0$ or $\bar{e}=0$. From (2.7), we obtain $c_{1,2}=a+b \pm 2 r$. Hence, we have at most four elements $\left\{a, b, c_{1}, c_{2}\right\}$ in such a polynomial $D(n)$-tuple, but we also have the following lemma.

Lemma 10. Let $a, b \in \mathbb{K}[X]$ such that $\operatorname{deg}(a)=\operatorname{deg}(b)=k \geq 3$ and $a b+n=r^{2}$. Then the set of the form $\{a, b, a+b+2 r, a+b-2 r\}$, which contains only polynomials of degree $k \geq 3$, is not a polynomial $D(n)$-quadruple.

Proof. The first part of the proof is analogue as the proof of Lemma 9, except that here $\operatorname{deg}(r)=k \geq 3$. Since in the case of polynomials of degree $k \geq 3$ the only possible triples are regular ones, we proved the lemma.

Therefore, the pair $\{a, b\}$ can be extended with at most one polynomial of degree $k$, where $k \geq 3$, (namely, one of $c_{1,2}$ ), so we proved Proposition 3 .

Example for this case is $D(X)$-triple $\left\{X^{3}-1, X^{3}+2 X^{2}+X-1,4 X^{3}+4 X^{2}+\right.$ $X-4\}$, from [8].

\section{GAP PRINCIPLE}

We will prove a gap principle for the degrees of the elements in a polynomial $D(n)$-quadruple. This result will be used in the proof of Theorem 1, together with the bounds from Section 2 and with the upper bound for the degree of the element in a polynomial $D(n)$-quadruple [8, Lemma 1], given in the following lemma. This bound was obtained for polynomials with integer coefficients but it also holds in $\mathbb{K}[X]$ with slightly different assumption on the degrees of polynomials in quadruple.

Lemma 11. Let $\{a, b, c, d\}$, where $\operatorname{deg}(a) \leq \operatorname{deg}(b) \leq \operatorname{deg}(c) \leq \operatorname{deg}(d)$, be a polynomial $D(n)$-quadruple with $n \in \mathbb{K}[X]$. Then

$$
\operatorname{deg}(d) \leq 7 \operatorname{deg}(a)+11 \operatorname{deg}(b)+15 \operatorname{deg}(c)+14 \operatorname{deg}(n)-4 .
$$


The proof of this lemma is based on the theory of function fields, precisely it is obtained by using Mason's inequality [16].

Now we will adjust the result from [7, Lemma 3], very similar as in the classical case for integers, to achieve the needed gap principle. We cannot use the gap principle from [7] and [8], because we do not have the relation " < " between elements of $\mathbb{K}[X]$.

Lemma 12. Let $\{a, b, c, d\}$, where $3 \leq \operatorname{deg}(a) \leq \operatorname{deg}(b) \leq \operatorname{deg}(c) \leq \operatorname{deg}(d)$, be $a$ polynomial $D(n)$-quadruple for linear $n \in \mathbb{K}[X]$. Then

$$
\operatorname{deg}(d) \geq \operatorname{deg}(b)+\operatorname{deg}(c)-2 .
$$

Proof. Applying Lemma 1 to the polynomial $D(n)$-triple $\{a, c, d\}$, we conclude that there exist $e, \bar{e} \in \mathbb{K}[X]$ such that, by (2.7), we have

$$
e \cdot \bar{e}=n^{2}(d-a-c-2 s)(d-a-c+2 s) .
$$

From (3.1), for $e=0$ or $\bar{e}=0$, we get

$$
d=a+c \pm 2 s .
$$

In this case $\operatorname{deg}(d) \leq \operatorname{deg}(c), \operatorname{sodeg}(d)=\operatorname{deg}(c)$.

Let $e, \bar{e} \in \mathbb{K}[X]$ be nonzero polynomials. Since $\operatorname{deg}(s)=\frac{\operatorname{deg}(a)+\operatorname{deg}(c)}{2} \leq \operatorname{deg}(c)$, from (3.1) we obtain

$$
\operatorname{deg}(e)+\operatorname{deg}(\bar{e}) \leq 2+2 \operatorname{deg}(d) .
$$

By (2.3) and (2.6), we conclude that the degree of one of the polynomials $e$ and $\bar{e}$ is equal to $\operatorname{deg}(a)+\operatorname{deg}(c)+\operatorname{deg}(d)$ and the degree of the other one is $\geq 0$. Hence, from (3.3) it follows that

$$
\operatorname{deg}(d) \geq \operatorname{deg}(a)+\operatorname{deg}(c)-2 .
$$

Analogously, applying Lemma 1 to the polynomial $D(n)$-triple $\{b, c, d\}$, we obtain that either

$$
d=b+c \pm 2 t
$$

or

$$
\operatorname{deg}(d) \geq \operatorname{deg}(b)+\operatorname{deg}(c)-2 .
$$

Assume that (3.5) holds. As for (3.2), we conclude that $\operatorname{deg}(d)=\operatorname{deg}(c)$. Therefore, (3.4) cannot hold at the same time. Otherwise, we would obtain $\operatorname{deg}(a) \leq 2$, a contradiction. Assume that (3.2) also holds. From (3.2) and (3.5) we obtain $a=b \pm 2 s \pm 2 t$. Therefore, if $\operatorname{deg}(a)<\operatorname{deg}(c)$ then $\operatorname{deg}(s)>\operatorname{deg}(a)$ and the polynomial on the right hand side of the previous equation has degree $>\operatorname{deg}(a)$ unless $\operatorname{deg}(a)=\operatorname{deg}(b)$ and $S= \pm T$. We conclude that

$$
\operatorname{deg}(a)=\operatorname{deg}(b)=\operatorname{deg}(c)=\operatorname{deg}(d)
$$

or

$$
\operatorname{deg}(a)=\operatorname{deg}(b)<\operatorname{deg}(c)=\operatorname{deg}(d) \text { and } S= \pm T .
$$


If (3.7) holds, then we have a polynomial $D(n)$-quadruple whose elements have degrees equal to $k$, where $k \geq 3$. This is not possible, according to Proposition 3 . Therefore, it holds (3.8). Since $\{a, c, d\}$ and $\{b, c, d\}$ are both regular triples (i.e. $\{a, b, c, d\}$ is a twice semi-regular $D(n)$-quadruple), by (2.13) we have $c d+n=$ $(c \pm s)^{2}$ and $c d+n=(c \pm t)^{2}$, where the signs \pm in last two equations are the same as signs in (3.2) and (3.5), respectively. If $c \pm s=c \pm t$, then we obtain $s^{2}=t^{2}$ so $a=b$, which is not possible. If $c \pm s=-c \mp t$, then $\pm s=-2 c \mp t$. Since (3.8) holds, the degree of the polynomial on the left hand side of the previous equation is $<\operatorname{deg}(c)$ and the polynomial on the right hand side of that equation has a degee equal to $\operatorname{deg}(c)$. This is not possible.

Assume that (3.6) holds. Then (3.2) can not hold, because otherwise we would have $\operatorname{deg}(b) \leq 2$, a contradiction. But, from (3.6) we obtain (3.4), which shows that this situation is indeed possible.

\section{Proof of Theorem 1}

Let $S=\left\{a_{1}, a_{2}, \ldots, a_{m}\right\}$, where $\operatorname{deg}\left(a_{1}\right) \leq \operatorname{deg}\left(a_{2}\right) \leq \ldots \leq \operatorname{deg}\left(a_{m}\right)$, be a polynomial $D(n)$ - $m$-tuple with $n \in \mathbb{K}[X]$ a linear polynomial. Since the product of each two elements from $S$ increased by $n$ is a square of a polynomial in $\mathbb{K}[X]$, it follows that if $S$ contains a polynomial of degree $\geq 1$, then it contains only polynomials of even or only polynomials of odd degree. We proved that in $S$ we have at most 1 nonzero constant. By Proposition 1, in $S$ there are at most 7 linear polynomials. By Proposition 2, the number of quadratic polynomials in $S$ is at most 4, and, by Proposition 3, in $S$ there are at most 3 polynomials of degree $k$ for every $k \geq 3$.

Assume that in $S$ there is a polynomial of degree $\geq 1$. Let us first consider the case where the degrees of all polynomials in $S$ are odd. We will combine the gaps between the degrees of the elements in $S$ with the upper bound on the degree of the element of a $D(n)$-quadruple. We assume that there are smallest possible gaps between the degrees of the elements in $S$. Then, there are possible the following bounds for the degrees:

$$
\begin{gathered}
\operatorname{deg}\left(a_{1}\right) \geq 1, \operatorname{deg}\left(a_{2}\right) \geq 1, \ldots, \operatorname{deg}\left(a_{7}\right) \geq 1, \\
\operatorname{deg}\left(a_{8}\right) \geq 3, \operatorname{deg}\left(a_{9}\right) \geq 3, \operatorname{deg}\left(a_{10}\right) \geq 3 .
\end{gathered}
$$

Applying Lemma 12 to the polynomial $D(n)$-quadruple $\left\{a_{8}, a_{9}, a_{10}, a_{11}\right\}$ gives $\operatorname{deg}\left(a_{11}\right) \geq 4$ and, since this degree is odd, we conclude that

$$
\operatorname{deg}\left(a_{11}\right) \geq 5
$$

If we continue in analogue way, we obtain

$$
\begin{array}{ll}
\operatorname{deg}\left(a_{12}\right) \geq 7, \quad & \operatorname{deg}\left(a_{13}\right) \geq 11, \quad \operatorname{deg}\left(a_{14}\right) \geq 17, \quad \operatorname{deg}\left(a_{15}\right) \geq 27, \\
\operatorname{deg}\left(a_{16}\right) \geq 43, & \operatorname{deg}\left(a_{17}\right) \geq 69, \quad \operatorname{deg}\left(a_{18}\right) \geq 111, \quad \operatorname{deg}\left(a_{19}\right) \geq 179, \ldots .
\end{array}
$$

We will separate the cases depending on the number of linear polynomials in $S$. Assume first that in $S$ we have at least three (and $\leq 7$ ) linear polynomials. For 
the quadruple $\left\{a_{1}, a_{2}, a_{3}, a_{m}\right\} \subseteq S$, where $\operatorname{deg}\left(a_{1}\right)=\operatorname{deg}\left(a_{2}\right)=\operatorname{deg}\left(a_{3}\right)=1$ and $\operatorname{deg}\left(a_{m}\right) \geq 1$, applying Lemma 11 , we get

$$
\operatorname{deg}\left(a_{m}\right) \leq 7+11+15+14-4=43 .
$$

Hence, in this case

$$
m \leq 16 .
$$

Assume next that in $S$ we have two linear polynomials. Now we observe the quadruple $\left\{a_{1}, a_{2}, a_{3}, a_{m}\right\} \subseteq S$, where $\operatorname{deg}\left(a_{1}\right)=\operatorname{deg}\left(a_{2}\right)=1$ and $\operatorname{deg}\left(a_{3}\right)=\mathrm{A}$, for $A \geq 3$ an odd positive integer. As before, we have

$$
\operatorname{deg}\left(a_{m}\right) \leq 7+11+15 A+14-4=15 A+28
$$

and

$$
\begin{array}{lll}
\operatorname{deg}\left(a_{4}\right) \geq \mathrm{A}, & \operatorname{deg}\left(a_{5}\right) \geq \mathrm{A}, & \operatorname{deg}\left(a_{6}\right) \geq 2 A-2, \ldots, \\
\operatorname{deg}\left(a_{10}\right) \geq 13 A-24, & \operatorname{deg}\left(a_{11}\right) \geq 21 A-40, & \operatorname{deg}\left(a_{12}\right) \geq 34 A-66, \\
\operatorname{deg}\left(a_{13}\right) \geq 55 A-108, & \operatorname{deg}\left(a_{14}\right) \geq 89 A-176, & \operatorname{deg}\left(a_{15}\right) \geq 144 A-286, \ldots,
\end{array}
$$

so we obtain $m \leq 13$.

Let in $S$ we have one linear polynomial, i.e. $\operatorname{deg}\left(a_{1}\right)=1, \operatorname{deg}\left(a_{2}\right)=A, \operatorname{deg}\left(a_{3}\right)=$ $B$, where $3 \leq A \leq B$ and $A, B$ are odd positive integers. We obtain

$$
\operatorname{deg}\left(a_{m}\right) \leq 7+11 A+15 B+14-4 \leq 26 B+17 .
$$

Further,

$$
\begin{array}{lll}
\operatorname{deg}\left(a_{4}\right) \geq B, & \operatorname{deg}\left(a_{5}\right) \geq 2 B-2, & \operatorname{deg}\left(a_{6}\right) \geq 3 B-4, \ldots, \\
\operatorname{deg}\left(a_{12}\right) \geq 55 B-108, & \operatorname{deg}\left(a_{13}\right) \geq 89 B-176, & \operatorname{deg}\left(a_{14}\right) \geq 144 B-286, \ldots,
\end{array}
$$

so $m \leq 13$.

Finally, suppose that $\operatorname{deg}\left(a_{1}\right)=A, \operatorname{deg}\left(a_{2}\right)=B, \operatorname{deg}\left(a_{3}\right)=C$ where $3 \leq A \leq B \leq$ $C$ and $A, B, C$ are odd positive integers. We get

$$
\operatorname{deg}\left(a_{m}\right) \leq 7 A+11 B+15 C+14-4 \leq 33 C+10
$$

and

$$
\begin{array}{ll}
\operatorname{deg}\left(a_{4}\right) \geq B+C-2, & \operatorname{deg}\left(a_{5}\right) \geq B+2 C-4, \ldots, \\
\operatorname{deg}\left(a_{12}\right) \geq 34 B+55 C-176, & \operatorname{deg}\left(a_{13}\right) \geq 55 B+89 C-286, \ldots,
\end{array}
$$

then $m \leq 12$. Hence, if $S$ contains only polynomials of odd degree then $m \leq 16$.

Let all polynomials in $S$ have even degree. Now we have

$$
\begin{gathered}
\operatorname{deg}\left(a_{1}\right) \geq 0, \\
\operatorname{deg}\left(a_{2}\right) \geq 2, \operatorname{deg}\left(a_{3}\right) \geq 2, \operatorname{deg}\left(a_{4}\right) \geq 2, \operatorname{deg}\left(a_{5}\right) \geq 2 \text { and } \\
\operatorname{deg}\left(a_{6}\right) \geq 4, \operatorname{deg}\left(a_{7}\right) \geq 4, \operatorname{deg}\left(a_{8}\right) \geq 4 .
\end{gathered}
$$

Applying Lemma 12 to the polynomial $D(n)$-quadruple $\left\{a_{6}, a_{7}, a_{8}, a_{9}\right\}$ we get

$$
\operatorname{deg}\left(a_{9}\right) \geq 6
$$


Analogously, it follows

$$
\begin{array}{ll}
\operatorname{deg}\left(a_{10}\right) \geq 8, \quad & \operatorname{deg}\left(a_{11}\right) \geq 12, \quad \operatorname{deg}\left(a_{12}\right) \geq 18, \quad \operatorname{deg}\left(a_{13}\right) \geq 28, \\
\operatorname{deg}\left(a_{14}\right) \geq 44, & \operatorname{deg}\left(a_{15}\right) \geq 70, \quad \operatorname{deg}\left(a_{16}\right) \geq 112, \quad \operatorname{deg}\left(a_{17}\right) \geq 180, \ldots
\end{array}
$$

Assume first that $\operatorname{deg}\left(a_{1}\right)=0, \operatorname{deg}\left(a_{2}\right)=A, \operatorname{deg}\left(a_{3}\right)=B$ where $2 \leq A \leq B$ and $A$, $B$ are even positive integers. If we apply Lemma 11 to a polynomial $D(n)$-quadruple $\left\{a_{1}, a_{2}, a_{3}, a_{m}\right\}$, it follows that

$$
\operatorname{deg}\left(a_{m}\right) \leq 0+11 A+15 B+14-4 \leq 26 B+10 .
$$

If $A=B=2$, then $m \leq 14$. If $B \geq 4$, then

$$
\begin{array}{lll}
\operatorname{deg}\left(a_{4}\right) \geq B, & \operatorname{deg}\left(a_{5}\right) \geq B, & \operatorname{deg}\left(a_{6}\right) \geq 2 B-2, \ldots, \\
\operatorname{deg}\left(a_{12}\right) \geq 34 B-66, & \operatorname{deg}\left(a_{13}\right) \geq 55 B-108, & \operatorname{deg}\left(a_{14}\right) \geq 89 B-176, \ldots,
\end{array}
$$

so we obtain that $m \leq 13$.

Suppose finally that $\operatorname{deg}\left(a_{1}\right)=A, \operatorname{deg}\left(a_{2}\right)=B, \operatorname{deg}\left(a_{3}\right)=C$, where $2 \leq A \leq B \leq C$ and $A, B, C$ are even positive integers. We have

$$
\operatorname{deg}\left(a_{m}\right) \leq 7 A+11 B+15 C+14-4 \leq 33 C+10 .
$$

If $A=B=C=2$ and

$$
\begin{array}{lll}
\operatorname{deg}\left(a_{4}\right) \geq C, & \operatorname{deg}\left(a_{5}\right)=D, & \operatorname{deg}\left(a_{6}\right) \geq D, \\
\operatorname{deg}\left(a_{7}\right) \geq D, & \operatorname{deg}\left(a_{8}\right) \geq 2 D-2, & \operatorname{deg}\left(a_{9}\right) \geq 3 D-4, \ldots, \\
\operatorname{deg}\left(a_{14}\right) \geq 34 D-66, & \operatorname{deg}\left(a_{15}\right) \geq 55 D-108, & \operatorname{deg}\left(a_{16}\right) \geq 89 D-176, \ldots,
\end{array}
$$

where $D \geq 4$ and $D$ is even positive integer, then $m \leq 15$. Also, $m \leq 13$ if $C \geq 4$. We conclude that the set $S$ has at most 15 polynomials of even degree. Therefore,

$$
L \leq 16 \text {. }
$$

\section{ACKNOWLEDGEMENT}

The authors would like to thank to Professor Andrej Dujella for introducing them to this problem.

\section{REFERENCES}

[1] I. G. Bashmakova, Diophantus of Alexandria, Arithmetics and the Book of Polygonal Numbers. Moscow: Nauka, 1974.

[2] A. Dujella, "On the size of diophantine $m$-tuples," Math. Proc. Camb. Philos. Soc., vol. 131, no. 1, pp. 23-33, 2002, doi: 10.1017/s0305004101005515.

[3] A. Dujella, "Bounds for the size of sets with the property $d(n)$," Glas. Mat., III. Ser., vol. 39, no. 2, pp. 199-205, 2004, doi: 10.3336/gm.39.2.01.

[4] A. Dujella, "There are only finitely many diophantine quintuples," J. Reine Angew. Math., vol. 556, pp. 183-214, 2004, doi: 10.1515/crll.2004.003.

[5] A. Dujella and C. Fuchs, "A polynomial variant of a problem of Diophantus and Euler," Rocky Mt. J. Math., vol. 33, no. 3, pp. 797-811, 2003, doi: 10.1216/rmjm/1181069929. 
[6] A. Dujella and C. Fuchs, "Complete solution of the polynomial version of a problem of Diophantus,” J. Number Theory, vol. 106, no. 2, pp. 326-344, 2004, doi: 10.1016/j.jnt.2003.12.011.

[7] A. Dujella, C. Fuchs, and R. F. Tichy, "Diophantine $m$-tuples for linear polynomials," Period. Math. Hung., vol. 45, no. 1-2, pp. 21-33, 2002.

[8] A. Dujella, C. Fuchs, and G. Walsh, "Diophantine $m$-tuples for linear polynomials. ii: Equal degrees," J. Number Theory, vol. 120, no. 2, pp. 213-228, 2006, doi: 10.1016/j.jnt.2005.12.005.

[9] A. Dujella and A. Jurasić, "On the size of sets in a polynomial variant of a problem of Diophantus," Int. J. Number Theory, vol. 6, no. 7, pp. 1449-1471, 2010, doi: 10.1142/s1793042110003575.

[10] A. Dujella and A. Jurasić, "Some diophantine triples and quadruples for quadratic polynomials," vol. 3, no. 2, pp. 1123-141, 2011.

[11] A. Dujella and F. Luca, "On a problem of Diophantus with polynomials," Rocky Mt. J. Math., vol. 37, no. 1, pp. 131-157, 2007.

[12] P. Gibbs, "Some rational diophantine sextuples," Glas. Mat., III. Ser., vol. 41, no. 2, pp. 195-203, 2006, doi: 10.3336/gm.41.2.02.

[13] B. W. Jones, "A variation on a problem of Davenport and Diophantus," Q. J. Math., Oxf. II. Ser., vol. 27, pp. 349-353, 1976.

[14] B. W. Jones, "A second variation on a problem of Davenport and Diophantus," Fibonacci Q., vol. 16, pp. 155-165, 1978.

[15] A. Jurasić, "Diophantine $m$-tuples for quadratic polynomials," Glas. Mat., III. Ser., vol. 46, no. 2, pp. 283-309, 2011, doi: 10.3336/gm.46.2.02.

[16] R. C. Mason, Diophantine equations over function fields. Cambridge: Cambridge University Press, 1984. doi: 10.1017/cbo9780511752490.

Authors' addresses

A. Filipin

University of Zagreb, Faculty of Civil Engineering, Fra Andrije Kačića-Miošića 26, 10000 Zagreb, Croatia

E-mail address: filipinegrad.hr

A. Jurasić

University of Rijeka, Department of Mathematics, Radmile Matejčić 2, 51000 Rijeka, Croatia

E-mail address: ajurasic@math.uniri.hr 